\begin{tabular}{|c|l|}
\hline Title & Growth of wurtzite GaP in InP/GaP core shell nanowires by selective area MOVPE \\
\hline Author(s) & Ishizaka, Fumiya; Hiraya, Y oshihiro; Tomioka, Katsuhiro; Fukui, Takashi \\
\hline Citation & $\begin{array}{l}\text { Journal of crystal growth, 411, 71-75 } \\
\text { https://doi.org/10.1016/.jcrysgro.2014.10.024 }\end{array}$ \\
\hline Issue Date & 2015-02-01 \\
\hline Doc URL & http://hdl.handle.net/2115/57932 \\
\hline Type & article(author version) \\
\hline File Information & 05_Ishizaka_JCG_Revised_manuscript.pdf \\
\hline
\end{tabular}

Instructions for use 


\title{
Growth of wurtzite GaP in InP/GaP core-shell nanowires by selective-area MOVPE
}

Fumiya Ishizaka $^{1 *}$, Yoshihiro Hiraya ${ }^{1}$, Katsuhiro Tomioka ${ }^{1,2}$, and Takashi Fukui ${ }^{1}$

${ }^{1}$ Graduate School of Information Science and Technology, and Research Center for Integrated Quantum Electronics (RCIQE), Hokkaido University, North 13 West 8, Sapporo 060-8628, Japan

${ }^{2}$ PRESTO, Japan Science and Technology Agency (JST), Honcho Kawaguchi, Saitama 332-0012, Japan

* Corresponding Author. Tel.: +81 11706 7173; fax: +81 117166004

E-mail address: ishizaka@rciqe.hokudai.ac.jp (F. Ishizaka)

\begin{abstract}
A GaP nanowire is promising from the viewpoint of device applications because when its crystal phase is changed from zinc blende (ZB) to wurtzite (WZ), its band gap changes from indirect to direct. GaP in the WZ phase is theoretically and experimentally shown to have the possibility of "green" emission. Here we report on the growth of WZ GaP in InP/GaP core-shell nanowires by selective-area metal-organic vapor-phase epitaxy (SA-MOVPE). WZ InP nanowires were used as a template for transferring the WZ structure to GaP. Transmission electron microscopy revealed that $\mathrm{WZ} \mathrm{GaP} \mathrm{was} \mathrm{grown} \mathrm{on} \mathrm{the} \mathrm{sidewalls} \mathrm{of} \mathrm{the} \mathrm{InP} \mathrm{core} \mathrm{in} \mathrm{the}$ lateral $<-211>$ direction and that ZB GaP was grown on the top of the InP core in the axial $<111>$ A direction. A growth model for the different crystal structures of the GaP shell is proposed from the viewpoint of the growth direction. The WZ structure is "transferred" from the InP core to the GaP shell only when GaP grows in the direction perpendicular to the $\mathrm{WZ}$ stacking direction of the InP core. This so-called “crystal structure transfer" can also be applied to p- and n-doped GaP and is therefore promising for fabricating WZ-GaP-based light-emitting diodes.
\end{abstract}




\section{Keywords}

A1. Nanostructures, A3. Metalorganic vapor phase epitaxy, A3. Selective epitaxy, B1. Nanomaterials, B2. Semiconducting III-V materials

\section{Introduction}

Semiconductor nanowires (NWs) have attracted considerable interest in recent years because of their potential applications in devices such as field-effect transistors [1,2], light-emitting diodes [3-6], and solar cells [7,8]. The most-surprising feature of nanowires is that they can be grown with a wurtzite (WZ) crystal structure by appropriately adjusting the growth conditions such as growth temperature, V/III ratio, and doping amount [9-16], even though they are stable in the zinc blende (ZB) phase of the bulk crystal. This crystal structure engineering opens up a new possibility for the device applications of nanowires because the crystal structure has a significant effect on the physical properties of semiconductor materials. Interestingly, band-structure calculations suggest that GaP in the WZ phase has a direct band gap [17-21], although that in the ZB phase has an indirect band gap. Recently, this suggestion has been experimentally confirmed by using WZ GaP nanowires fabricated by vapor-liquid-solid (VLS) growth, suggesting the direct-band-gap transition at $2.09 \mathrm{eV}$ [14]. This direct-band-gap WZ GaP may increase the efficiency of green light-emitting diodes and close the "green gap" issue.

We have grown III-V compound semiconductor nanowires by selective-area metal-organic vapor-phase epitaxy (SA-MOVPE) [1,4,8-11,22-26]. The crystal structures of InP nanowires can be controlled by adjusting the growth conditions of SA-MOVPE, and pure WZ InP nanowires can be obtained at high growth temperature and low V/III ratio [9,10]. However, this type of control is not yet possible with other III-V compounds, such as GaP, GaAs, and InAs. To promote the crystal-phase change of those compounds, it is necessary to transfer the WZ structure from the core to the shell. We have already reported pure WZ InAs nanotubes 
based on InP/InAs core-shell nanowires, in which the WZ structure of the InP core is transferred to the InAs shell [22]. In this study, we demonstrate the growth of WZ GaP by using $\mathrm{WZ}$ InP nanowires as a template for transferring the $\mathrm{WZ}$ structure to $\mathrm{GaP}$, and propose a possible growth model for the crystal structure transfer. InP was chosen as a template because the WZ structures can be easily obtained. From the calculation, the energy difference between the $\mathrm{WZ}$ and $\mathrm{ZB}$ structures of InP is a minimum in the case of typical III-V compounds including aluminum, gallium, and indium as group-III elements and phosphorus, arsenic, and antimony as group-V elements [27].

\section{Experimental procedure}

As for the process for fabricating the nanowires, a 25-nm-thick $\mathrm{SiO}_{2}$ film was first deposited on an InP (111)A substrate by plasma sputtering, and hexagonal-opening patterns were defined by electron-beam (EB) lithography and wet chemical etching using buffered hydrofluoric acid (BHF). The $\mathrm{SiO}_{2}$ patterns were designed to be a periodic array of openings with a diameter of $90 \mathrm{~nm}$ and a pitch of $400 \mathrm{~nm}$. The SA-MOVPE of nanowires was carried out in a horizontal low-pressure MOVPE system using trimethylgallium (TMGa), trimethylindium (TMIn), and tertiarybutylphosphine (TBP) as source materials. The partial pressures of TMIn and TBP were respectively $2.7 \times 10^{-6}$ and $4.9 \times 10^{-5}$ atm for InP core growth, and the partial pressures of TMGa and TBP were respectively $4.8 \times 10^{-7}$ and $3.1 \times 10^{-4}$ for GaP shell growth. Prior to the growth, the native oxide on the openings was removed by thermal cleaning at $600^{\circ} \mathrm{C}$ under a hydrogen and TBP ambient. WZ InP nanowires were grown for $20 \mathrm{~min}$ at $660^{\circ} \mathrm{C}$ with a V/III ratio of 18 , which is the typical condition for growing WZ structures [9,10]. GaP shells were then grown for $3 \mathrm{~min}$ at $600^{\circ} \mathrm{C}$ with a V/III ratio of 646 . This low-temperature and high-V/III-ratio condition was chosen to promote lateral growth of the shell $[23,28]$. The grown structures were characterized by scanning electron microscope (SEM) and transmission electron microscope (TEM). As for the TEM characterization, the electron-beam projection 
was parallel to the $<-110>$ direction.

\section{Results and discussion}

\section{1. Structure of grown InP/GaP core-shell nanowires}

Figure 1 shows a $15^{\circ}$-tilted SEM image of the grown InP/GaP core-shell nanowires, which have hexagonal $\{10-10\}$ vertical sidewall facets with average diameter and height of $100 \mathrm{~nm}$ and $1.6 \mu \mathrm{m}$, respectively. Sidewalls of InP nanowires with WZ structures are typically $\{10-10\}$ facets (which correspond to $\{-211\}$ facets in cubic ZB notation), but those with ZB structures are $\{-110\}$ facets. SEM studies revealed that growth of the GaP shell was always non-uniform and that three-dimensional growth was partially observed, probably due to the large lattice mismatch of the InP/GaP material system (7.1\%). In the case of InP/InAs core-shell nanowires (with lattice mismatch of 3.2\%) [22], a uniform InAs shell can be obtained at low growth temperature and a small supply of group-III precursors. Accordingly, to improve the uniformity of the shell, it is necessary to further optimize the growth condition and/or the buffer layer between the core and shell.

The crystal structure of core-shell nanowires was clarified by high-resolution TEM. Figure 2(a) shows a TEM image of the InP/GaP interface at the sidewall of a core-shell nanowire. As shown in Figs. 2(b) and 2(c), the TEM image of the InP core and the corresponding fast-Fourier-transform (FFT) image revealed that the template InP nanowires had a WZ structure with a few stacking faults per micrometer. From the magnified TEM image and the corresponding FFT image in Figs. 2(d) and 2(e), it is confirmed that WZ GaP was grown laterally on the sidewalls of the WZ InP nanowires. The thickness of the GaP shell was about 5 nm. The spot splitting in the FFT image was caused by two different lattice spacings, which indicates the existence of the GaP shell. The GaP shell was also confirmed by energy-dispersive X-ray spectroscopy (EDX) line scans along the lateral direction of an InP/GaP core-shell nanowire, with a corresponding high-angle annular dark-field (HAADF) 
image, as shown in Fig. 2(f). Misfit dislocations are also observed in Figs. 2(a) and 2(d). The correlation between misfit dislocations and strain at the InP/GaP interface was investigated. Under the assumption of complete relaxation of the InP/GaP core-shell nanowires, the spacing between misfit dislocations is calculated from its lattice mismatch of $7.1 \%$ to be $4.4 \mathrm{~nm}$ by using the ZB lattice parameters of InP and GaP. The measured average spacing was $10.6 \mathrm{~nm}$, which differs from the calculated value. This result means that $40 \%$ of interface stress was accommodated by misfit dislocations and the rest was maintained in the InP/GaP interface.

Figure 3(a) shows a high-resolution TEM image of the top of an InP/GaP core-shell nanowire. The EDX line scans of the GaP top shell in Fig. 3(b) shows that indium at a composition of $20 \%$ was incorporated into GaP, probably due to surface segregation of indium during growth of GaP. Magnified TEM and FFT images of GaP grown on the top of an InP nanowire are shown in Figs. 3(c) and (d), which indicate that GaP has a ZB structure. A magnified TEM image of the interface of $\mathrm{WZ}$ InP and ZB GaP is shown in Fig. 3(e). The crystal structures of nanowires completely changed from WZ to ZB after growth of a monolayer of GaP, and stacking faults and twins did not appear near the interface except for the phase transition boundary. In other words, when GaP growth started in the $<111>\mathrm{A}$ direction, the growth mode immediately changed to the same situation as the selective-area growth of GaP on a planar (111)A substrate. In growth of GaP by SA-MOVPE on GaP(111)A and $\mathrm{B}$ substrates [25], although hexagonal nanowires are grown in the $<111>\mathrm{B}$ direction, tetrahedral structures with (111)B facets are formed in the $<111>$ A direction. In addition, these tetrahedral GaP structures formed on the (111)A plane are thought to have ZB phase because the corners of all tetrahedrons are pointed in one direction [24]. In the present study, tetrahedral-like GaP structures, in which (111)B facets partially appeared, were formed on the top of InP nanowires and had ZB phase. Note that the InP nanowires, as well as the GaP top shell, were grown in the $<111>$ A direction. The present result is consistent with our previous study on growth of GaP by SA-MOVPE. 


\section{2. Different crystal structures of GaP shell}

Crystal structures of InP/GaP core-shell nanowires are summarized schematically in Fig. 4(a). The reason that different crystal structures were grown on the top and side of the InP nanowires is discussed in the following from the viewpoint of the stacking direction of a WZ InP template. Atomistic models for structural transition in InP/GaP core-shell nanowires are shown in Figure 4(b). In the axial $<111>$ A direction, the stacking sequences of the WZ phase (ABAB...) and the ZB phase (ABCABC...) are geometrically variable to each other without introducing mis-coordinated atoms because the growth direction of the top shell is the same as the WZ stacking direction. The crystal structure of the GaP top shell can therefore be changed in accordance with the materials of the shell and growth conditions. In our previous study on InGaP nanowires on InP(111)A substrates [26], the crystal phase transition from WZ to ZB occurred when a small amount of gallium was added to WZ InP nanowires, indicating that GaP has a strong tendency to form ZB phase. In addition, in this study, the GaP shell was grown under the condition of low growth temperature and high V/III ratio, at which ZB structures are likely to form $[13,28]$. Hence, ZB GaP was formed on the top of the InP nanowires.

In the lateral <-211> direction, however, the WZ stacking sequence “ABAB...” is strongly fixed because the growth direction of the shell is perpendicular to the WZ stacking direction. In this situation, crystal structures can be transferred in accordance with the energy balance for the core and shell materials. Major factors affecting this balance are the defect-formation energy and the energy difference between the WZ and ZB structures (hereafter, "WZ-ZB energy difference”), which was calculated by Yeh et al. [27] When the WZ-ZB energy difference is larger than the defect-formation energy, ZB structures are formed by introducing many misfit dislocations between core and shell layers. When the WZ-ZB energy difference is small enough, however, WZ structures are maintained during shell growth. In this study, according to the TEM image in Fig. 2(d), the GaP side shell was grown coherently with a few 
monolayers before the misfit dislocations were introduced, and this coherent growth of the shell indicates a relatively large defect-formation energy. The WZ-ZB energy difference was therefore probably much smaller than the defect-formation energy, resulting in the sequence “ABAB...” being transferred from the InP core to the GaP shell regardless of the growth conditions. The key requirements concerning this technique for transferring the crystal structure are the twofold: first, growth direction is perpendicular to the WZ stacking direction; second, the WZ-ZB energy difference is smaller than the defect-formation energy.

\section{Summary}

We demonstrated the growth of WZ GaP in InP/GaP core-shell nanowires by SA-MOVPE, in which the $\mathrm{WZ}$ InP nanowires were used as a template for transferring the $\mathrm{WZ}$ structure to the GaP shell. TEM and FFT analyses revealed that WZ GaP was grown on the sidewalls of the InP core in the lateral $<-211>$ direction and that $\mathrm{ZB} \mathrm{GaP}$ was also grown on the top of the InP core in the axial $<111>$ A direction. Next, we showed a growth model for the different crystal structures of GaP. The "ABAB...” stacking sequence of WZ phase is strongly fixed in the $<-211>$ direction, while both the WZ and ZB phases are geometrically possible in the $<111>\mathrm{A}$ direction. A WZ structure is transferred from the InP core to the GaP shell in the <-211> direction because the WZ-ZB energy difference is relatively small. This crystal structure transfer can be applied to $\mathrm{p}$ - and n-doped $\mathrm{GaP}$ and is therefore promising for fabricating WZ-GaP-based light-emitting diodes.

\section{Acknowledgements}

The authors would like to thank Professor Junichi Motohisa, Professor Shinjiro Hara, and Dr. Keitaro Ikejiri for their valuable discussions as well as Dr. Masatoshi Yoshimura and Mr. Eiji Nakai for their support with the experiments. This work was financially supported by a Grant-in-Aid for Specially Promoted Scientific Research provided by the Ministry of 
Education, Culture, Sports, Science, and Technology (MEXT), Japan.

\section{References}

[1] K. Tomioka, M. Yoshimura, T. Fukui, A III-V nanowire channel on silicon for high-performance vertical transistors, Nature 488 (2012) 189.

[2] H. T. Ng, J. Han, T. Yamada, P. Nguyen, Y. P. Chen, M. Meyyappan, Single Crystal Nanowire Vertical Surround-Gate Field-Effect Transistor, Nano Lett. 4 (2004) 1247.

[3] E. Dimakis, U. Jahn, M. Ramsteiner, A. Tahraoui, J. Grandal, X. Kong, O. Marquardt, A. Trampert, H. Riechert, L. Geelhaar, Coaxial Multishell (In,Ga)As/GaAs Nanowires for Near-Infrared Emission on Si Substrates, Nano Lett. 14 (2014) 2604.

[4] K. Tomioka, J. Motohisa, S. Hara, K. Hiruma, T. Fukui, GaAs/AlGaAs Core Multishell Nanowire-Based Light-Emitting Diodes on Si, Nano Lett. 10 (2010) 1639.

[5] C. P. T. Svensson, T. Mårtensson, J. Trägårdh, C. Larsson, M. Rask, D. Hessman, L. Samuelson, J. Ohlsson, Monolithic GaAs/InGaP nanowire light emitting diodes on silicon, Nanotechnology 19 (2008) 305201.

[6] K. Hiruma, M. Yazawa, T. Katsuyama, K. Ogawa, K. Haraguchi, M. Koguchi, H. Kakibayashi, Growth and optical properties of nanometer-scale GaAs and InAs Growth and optical whiskers, J. Appl. Phys. 77 (1995) 447.

[7] J. Wallentin, N. Anttu, D. Asoli, M. Huffman, I. Åberg, M. H. Magnusson, G. Siefer, P. Fuss-Kailuweit, F. Dimroth, B. Witzigmann, H. W. Xu, L. Samuelson, K. Deppert, M. T. Borgström, InP Nanowire Array Solar Cells Achieving 13.8\% Efficiency by Exceeding the Ray Optics Limit, Science 339 (2013) 1057.

[8] M. Yoshimura, E. Nakai, K. Tomioka, T. Fukui, Indium tin oxide and indium phosphide heterojunction nanowire array solar cells, Appl. Phys. Lett. 103 (2013) 243111.

[9] Y. Kitauchi, Y. Kobayashi, K. Tomioka, S. Hara, K. Hiruma, T. Fukui, J. Motohisa, Structural Transition in Indium Phosphide Nanowires, Nano Lett. 10 (2010) 1699.

[10] K. Ikejiri, Y. Kitauchi, K. Tomioka, J. Motohisa, T. Fukui, Zinc Blende and Wurtzite Crystal Phase Mixing and Transition in Indium Phosphide Nanowires, Nano Lett. 11 (2011) 4314. 
[11] K. Ikejiri, F. Ishizaka, K. Tomioka, T. Fukui, Bidirectional Growth of Indium Phosphide Nanowires, Nano Lett. 12 (2012) 4770.

[12] Q. Gao, D. Saxena, F. Wang, L. Fu, S. Mokkapati, Y. Guo, L. Li, J. W.-Leung, P. Caroff, H. H. Tan, C. Jagadish, Selective-Area Epitaxy of Pure Wurtzite InP Nanowires: High Quantum Efficiency and Room-Temperature Lasing, Nano Lett. 14 (2014) 5206.

[13] R. E. Algra, M. Hocevar, M. A. Verheijen, I. Zardo, G. G. W. Immink, W. J. P. van Enckevort, G. Abstreiter, L. P. Kouwenhoven, E. Vlieg, E. P. A. M. Bakkers, Crystal Structure Transfer in Core/Shell Nanowires, Nano Lett. 11 (2011) 1690.

[14] S. Assali, I. Zardo, S. Plissard, D. Kriegner, M. A. Verheijen, G. Bauer, A. Meijerink, A. Belabbes, F. Bechstedt, J. E. M. Haverkort, E. P. A. M. Bakkers, Direct Band Gap Wurtzite Gallium Phosphide Nanowires, Nano Lett. 13 (2013) 1559.

[15] A. Berg, S. Lehmann, N. Vainorius, A. Gustafsson, M.-E. Pistol, L. R. Wallenberg, L. Samuelson, M. T. Borgström, Growth and characterization of wurtzite GaP nanowires with control over axial and radial growth by use of $\mathrm{HCl}$ in-situ etching, J. Cryst. Growth 386 (2014) 47.

[16] E. Husanu, D. Ercolani, M. Gemmi, L. Sorba, Growth of defect-free GaP nanowires, Nanotechnology 25 (2014) 205601.

[17] M. Murayama, T. Nakayama, Chemical trend of band offsets at wurtzite/zinc-blende heterocrystalline semiconductor interfaces, Phys. Rev. B 49 (1994) 4710.

[18] C.-Y. Yeh, S.-H. Wei, A. Zunger, Relationships between the band gaps of the zinc-blende and wurtzite modifications of semiconductors, Phys. Rev. B 50 (1994) 2715.

[19] A. De, C. E. Pryor, Predicted band structures of III-V semiconductors in the wurtzite phase, Phys. Rev. B 81 (2010) 155210.

[20] A. Belabbes, C. Panse, J. Furthmüller, F. Bechstedt, Electronic bands of III-V semiconductor polytypes and their alignment, Phys. Rev. B 86 (2012) 075208.

[21] T. Akiyama, K. Nakamura, T. Ito, A. J. Freeman, Electronic bands and excited states of III-V semiconductor polytypes with screened-exchange density functional calculations, Appl. Phys. Lett. 104 (2014) 132101.

[22] P. Mohan, J. Motohisa, T. Fukui, Realization of conductive InAs nanotubes based on 
lattice-mismatched InP/InAs core-shell nanowires, Appl. Phys. Lett. 88 (2006) 013110.

[23] P. Mohan, J. Motohisa, T. Fukui, Controlled growth of highly uniform, axial/radial direction-defined, individually addressable InP nanowire arrays, Nanotechnology 16 (2005) 2903.

[24] H. Yoshida, K. Ikejiri, T. Sato, S. Hara, K. Hiruma, J. Motohisa, T. Fukui, Analysis of twin defects in GaAs nanowires and tetrahedra and their correlation to GaAs(111)B surface reconstructions in selective-area metal organic vapour-phase epitaxy, J. Cryst. Growth 312 (2009) 52.

[25] K. Tomioka, K. Ikejiri, T. Tanaka, J. Motohisa, S. Hara, K. Hiruma, T. Fukui, Selective-area growth of III-V nanowires and their applications, J. Mater. Res. 26 (2011) 2127.

[26] F. Ishizaka, K. Ikejiri, K. Tomioka, T. Fukui, Indium-Rich InGaP Nanowires Formed on InP (111)A Substrates by Selective-Area Metal Organic Vapor Phase Epitaxy, Jpn. J. Appl. Phys. 52 (2013) 04CH05.

[27] C.-Y. Yeh, Z.W. Lu, S. Froyen, A. Zunger, Zinc-blende-wurtzite polytypism in semiconductors, Phys. Rev. B 46 (1992) 10086.

[28] M. A. Verheijen, R. E. Algra, M. T. Borgström, G. Immink, E. Sourty, W. J. P. van Enckevort, E. Vlieg, E. P. A. M. Bakkers, Three-Dimensional Morphology of GaPGaAs Nanowires Revealed by Transmission Electron Microscopy Tomography, Nano Lett. 7 (2007) 3051. 


\section{Figure captions}

Figure 1: $15^{\circ}$-tilted SEM image of InP/GaP core-shell nanowires, which have $\{10-10\}$ side facets. The inset shows a magnified image of one nanowire.

Figure 2: Crystal structures at the side of an InP/GaP core-shell nanowire. (a) TEM image of the InP/GaP interface. (b) and (c) Magnified TEM image of InP core and the corresponding FFT image, showing a pure WZ structure. (d) and (e) Magnified TEM image at the InP/GaP interface and the corresponding FFT image, showing the existence of WZ GaP. (f) EDX line scans along the lateral direction of a core-shell nanowire and corresponding HAADF image. Black arrows in (a) and (d) indicate the positions of misfit dislocations.

Figure 3: Crystal structures at the top of an InP/GaP core-shell nanowire. (a) Overall TEM image at the top of the core-shell nanowire. (b) EDX line scans of the GaP top shell along the lateral direction and corresponding HAADF image. (c) and (d) Magnified TEM image of GaP top shell and the corresponding FFT image, showing a pure ZB structure. (e) Magnified TEM image at the interface of $\mathrm{WZ}$ and $\mathrm{ZB}$ phases.

Figure 4: (a) Schematic illustration of grown crystal structures of $\mathrm{InP} / \mathrm{GaP}$ core-shell nanowires. (b) Atomistic models for structural transition of the GaP shell. The stacking sequence of the WZ phase of "ABAB...” is strongly fixed in the $<-211>$ A direction (fixed stacking axis), while both $\mathrm{WZ}$ and $\mathrm{ZB}$ phases are geometrically possible in the $<111>\mathrm{A}$ direction (variable stacking axis). 
Figure 1

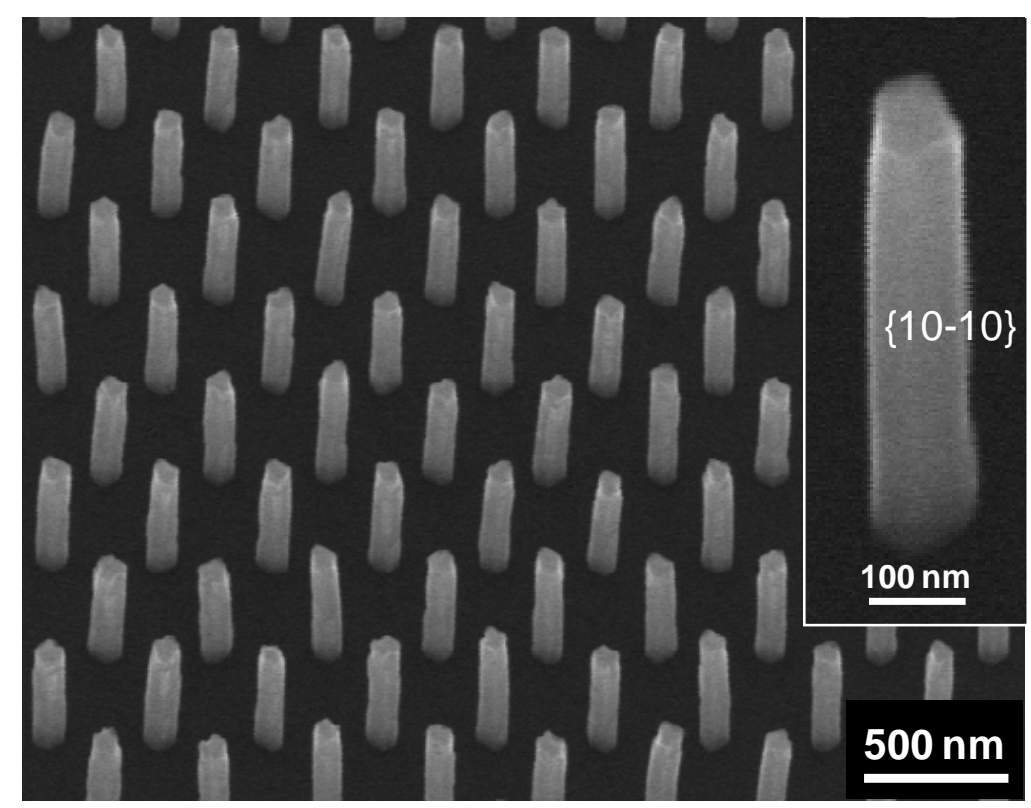


Figure 2
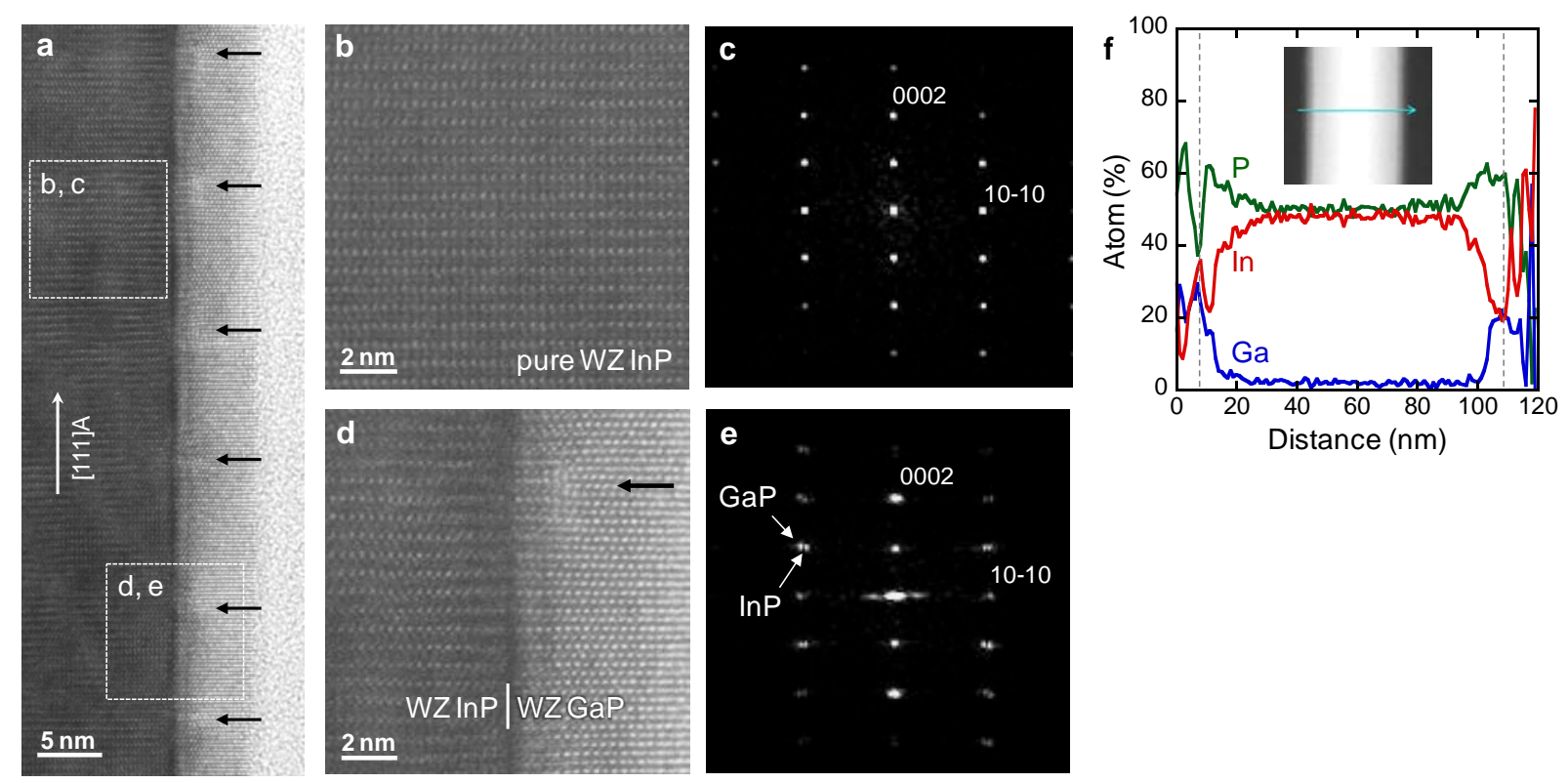
Figure 3
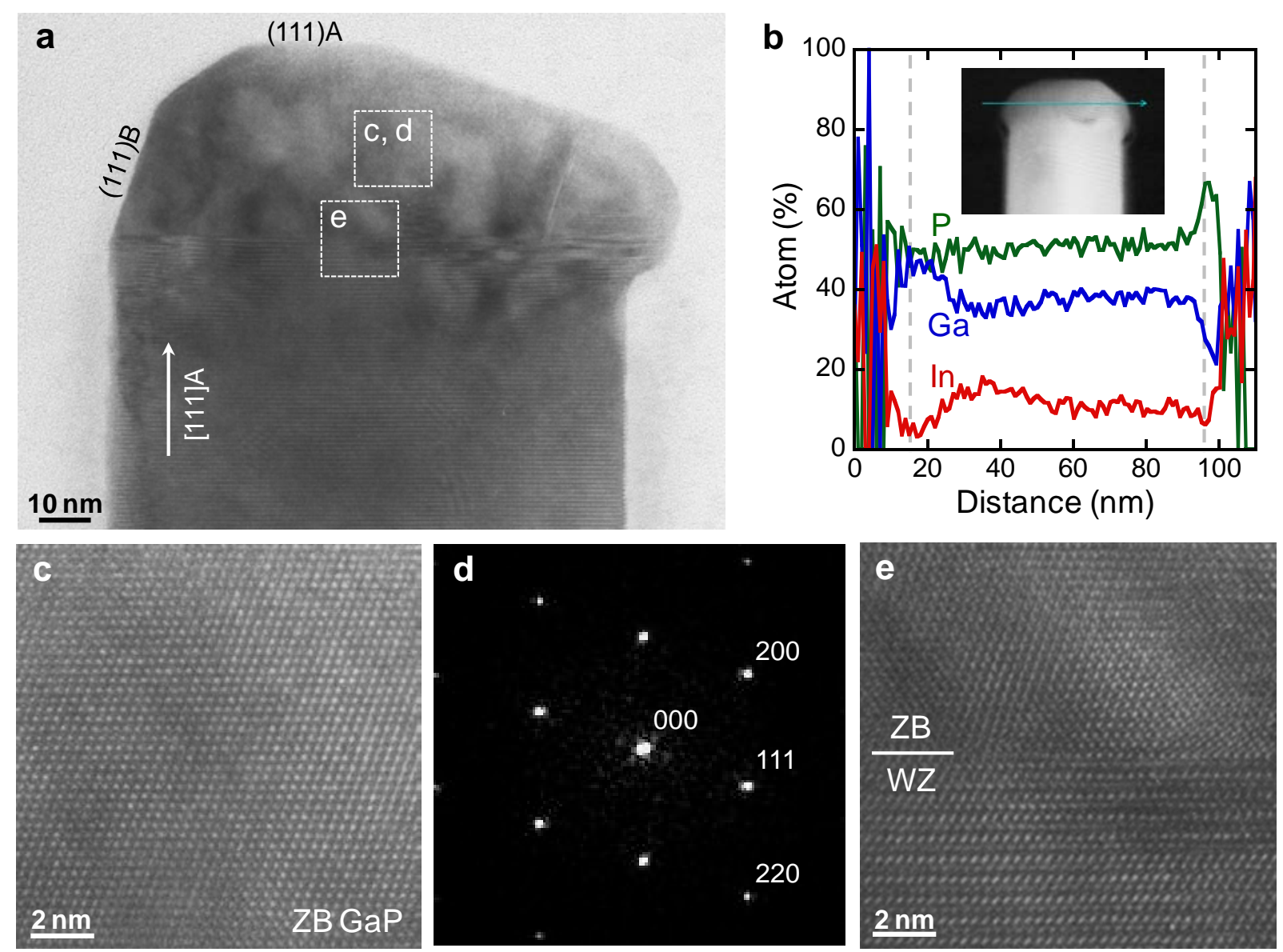
Figure 4
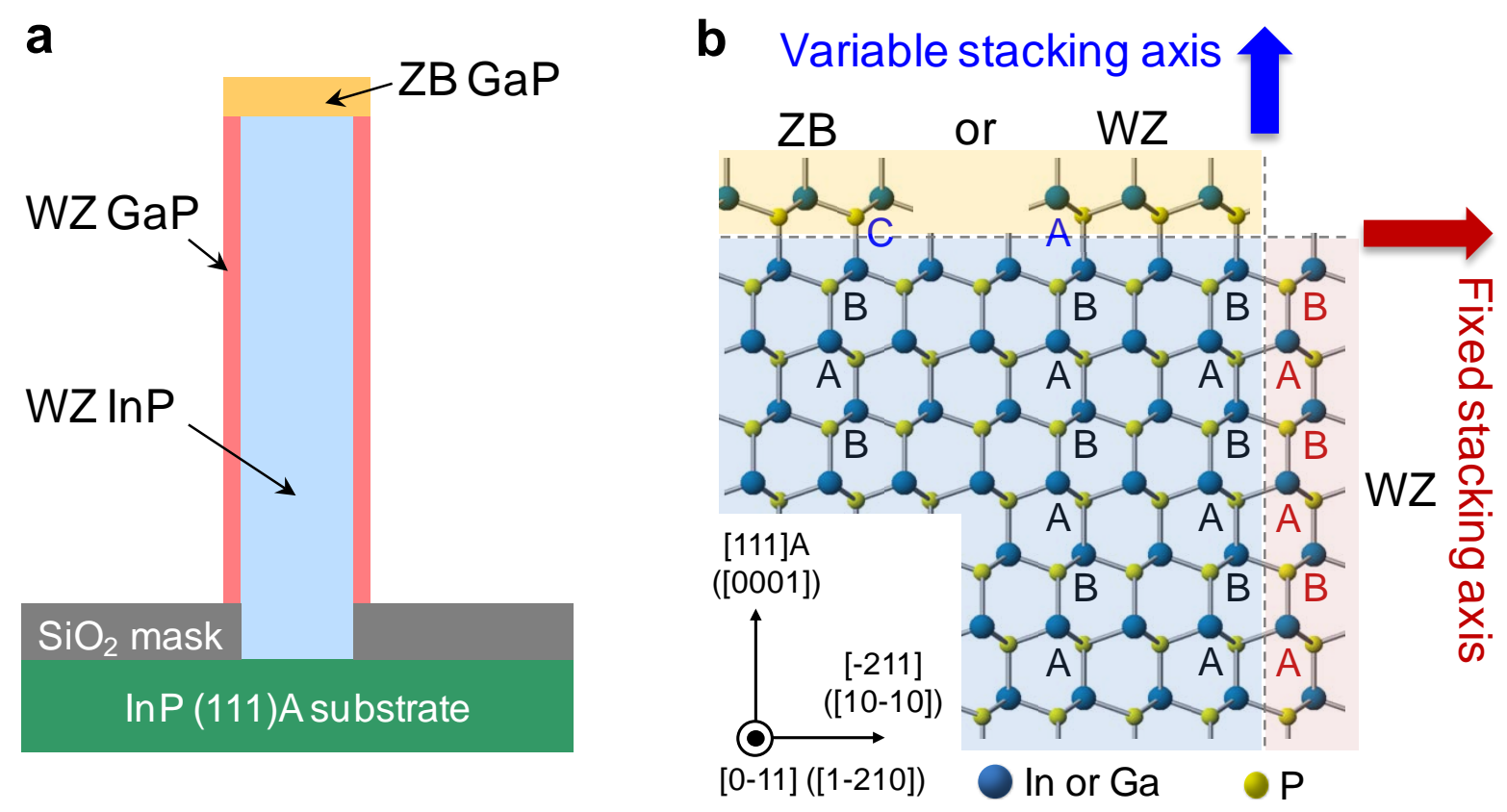